\title{
A New Subclass of Analytic Functions Involving Al-Oboudi Differential Operator
}

\section{Sevtap Sümer Eker and H. Özlem Güney}

Department of Mathematics, Faculty of Science and Letters, Dicle University, 21280 Diyarbakir, Turkey

Correspondence should be addressed to Sevtap Sümer Eker, sevtaps@dicle.edu.tr

Received 25 September 2007; Accepted 4 February 2008

Recommended by Jozsef Szabados

The main object of this paper is to introduce and investigate a new subclass of normalized analytic functions in the open unit disc $\mathbb{U}$ which is defined by Al-Oboudi differential operator. Coefficient inequalities, extreme points, and integral means inequalities for fractional derivative for this class are given.

Copyright (c) 2008 S. Sümer Eker and H. Özlem Güney. This is an open access article distributed under the Creative Commons Attribution License, which permits unrestricted use, distribution, and reproduction in any medium, provided the original work is properly cited.

\section{Introduction and definitions}

Let $\mathcal{A}$ denote the class of functions $f$ normalized by

$$
f(z)=z+\sum_{j=2}^{\infty} a_{j} z^{j}
$$

which are analytic in the open unit disc $\mathbb{U}=\{z:|z|<1\}$.

For $f \in \mathcal{A}$, Al-Oboudi [1] introduced the following operator:

$$
\begin{gathered}
D^{0} f(z)=f(z), \\
D^{1} f(z)=(1-\delta) f(z)+\delta z f^{\prime}(z)=D_{\delta} f(z), \quad \delta \geq 0 \\
D^{n} f(z)=D_{\delta}\left(D^{n-1} f(z)\right), \quad(n \in \mathbb{N}=1,2,3, \ldots) .
\end{gathered}
$$

If $f$ is given by (1.1), then from (1.3) and (1.4) we see that

$$
D^{n} f(z)=z+\sum_{j=2}^{\infty}[1+(j-1) \delta]^{n} a_{j} z^{j}, \quad\left(n \in \mathbb{N}_{0}=\mathbb{N} \cup\{0\}\right) .
$$

When $\delta=1$, we get Sălăgean differential operator [2]. 
Definition 1.1. Let $\mathcal{S}_{m, n, \delta}(\alpha)$ denote the subclass of $\mathcal{A}$ consisting of functions $f$ which satisfy the inequality

$$
\operatorname{Re}\left(\frac{D^{m} f(z)}{D^{n} f(z)}\right)>\alpha
$$

for some $0 \leq \alpha<1, m \in \mathbb{N}, n \in \mathbb{N}_{0}$, and all $z \in \mathbb{U}$.

The object of the present paper is to investigate the coefficient bounds, extreme points, and integral mean inequalities for fractional derivatives of functions belonging to the class $\mathcal{S}_{m, n, \delta}(\alpha)$.

\section{Coefficient inequalities}

Our first theorem gives a sufficient condition for $f \in \mathcal{A}$ to belong to the class $\mathcal{S}_{m, n, \delta}(\alpha)$.

Theorem 2.1. Let $f(z) \in \mathcal{A}$ satisfy

$$
\sum_{j=2}^{\infty} \Psi(m, n, j, \delta, \alpha)\left|a_{j}\right| \leq 2(1-\alpha),
$$

where

$$
\Psi(m, n, j, \delta, \alpha)=\left|[1+(j-1) \delta]^{m}-(1+\alpha)[1+(j-1) \delta]^{n}\right|+[1+(j-1) \delta]^{m}+(1-\alpha)[1+(j-1) \delta]^{n}
$$

for some $\alpha(0 \leq \alpha<1), m \in \mathbb{N}, n \in \mathbb{N}_{0}, \delta(\delta \geq 0)$. Then $f(z) \in \mathcal{S}_{m, n, \delta}(\alpha)$.

Proof. Suppose that (2.1) is true for $\alpha(0 \leq \alpha<1), m \in \mathbb{N}, n \in \mathbb{N}_{0}$, and $\delta(\delta \geq 0)$. For $f(z) \in \mathcal{A}$, define the function $F(z)$ by

$$
F(z)=\frac{D^{m} f(z)}{D^{n} f(z)}-\alpha
$$

It suffices to show that

$$
\left|\frac{F(z)-1}{F(z)+1}\right|<1 \quad(z \in \mathbb{U})
$$

We note that

$$
\begin{aligned}
\left|\frac{F(z)-1}{F(z)+1}\right| & =\left|\frac{D^{m} f(z) / D^{n} f(z)-\alpha-1}{D^{m} f(z) / D^{n} f(z)-\alpha+1}\right| \\
& =\left|\frac{D^{m} f(z)-(1+\alpha) D^{n} f(z)}{D^{m} f(z)+(1-\alpha) D^{n} f(z)}\right| \\
& =\left|\frac{\alpha-\sum_{j=2}^{\infty}\left([1+(j-1) \delta]^{m}-(1+\alpha)[1+(j-1) \delta]^{n}\right) a_{j} z^{j-1}}{(2-\alpha)+\sum_{j=2}^{\infty}\left([1+(j-1) \delta]^{m}+(1-\alpha)[1+(j-1) \delta]^{n}\right) a_{j} z^{j-1}}\right| \\
& \leq \frac{\alpha+\sum_{j=2}^{\infty}\left|[1+(j-1) \delta]^{m}-(1+\alpha)[1+(j-1) \delta]^{n}\right|\left|a_{j}\right||z|^{j-1}}{(2-\alpha)-\sum_{j=2}^{\infty}\left([1+(j-1) \delta]^{m}+(1-\alpha)[1+(j-1) \delta]^{n}\right)\left|a_{j}\right||z|^{j-1}} \\
& <\frac{\alpha+\sum_{j=2}^{\infty}\left|[1+(j-1) \delta]^{m}-(1+\alpha)[1+(j-1) \delta]^{n}\right|\left|a_{j}\right|}{(2-\alpha)-\sum_{j=2}^{\infty}\left([1+(j-1) \delta]^{m}+(1-\alpha)[1+(j-1) \delta]^{n}\right)\left|a_{j}\right|} .
\end{aligned}
$$


The last expression is bounded above by 1 if

$$
\begin{aligned}
& \alpha+\sum_{j=2}^{\infty}\left|[1+(j-1) \delta]^{m}-(1+\alpha)[1+(j-1) \delta]^{n}\right|\left|a_{j}\right| \\
& \quad \leq(2-\alpha)-\sum_{j=2}^{\infty}\left([1+(j-1) \delta]^{m}+(1-\alpha)[1+(j-1) \delta]^{n}\right)\left|a_{j}\right|
\end{aligned}
$$

which is equivalent to condition (2.1). This completes the proof of Theorem 2.1.

Example 2.2. The function $f(z)$ given by

$$
f(z)=z+\sum_{j=2}^{\infty} \frac{2(2+\gamma)(1-\alpha) \epsilon_{j}}{(j+\gamma)(j+1+\gamma) \Psi(m, n, j, \delta, \alpha)} z^{j}
$$

belongs to the class $S_{m, n, \delta}(\alpha)$ for $\gamma>-2,0 \leq \alpha<1, \epsilon_{j} \in \mathbb{C}$, and $\left|\epsilon_{j}\right|=1$.

We now derive the coefficient inequalities for $f(z)$ belonging to the class $\mathcal{S}_{m, n, \delta}(\alpha)$.

Theorem 2.3. If $f(z) \in \mathcal{S}_{m, n, \delta}(\alpha)$, then for $k \geq 2$,

$$
\begin{aligned}
\left|a_{k}\right| \leq \frac{\beta}{\left|v_{k}\right|}\{1 & +\beta \sum_{j=2}^{k-1} \frac{[1+(j-1) \delta]^{n}}{\left|v_{j}\right|}+\beta^{2} \sum_{j_{2}>j_{1}}^{k-1} \sum_{j_{1}=2}^{k-2} \frac{\left(\left[1+\left(j_{1}-1\right) \delta\right]\left[1+\left(j_{2}-1\right) \delta\right]\right)^{n}}{\left|v_{j_{1}} v_{j_{2}}\right|} \\
& +\beta^{3} \sum_{j_{3}>j_{2}}^{k-1} \sum_{j_{2}>j_{1}}^{k-2} \sum_{j_{1}=2}^{k-3} \frac{\left(\left[1+\left(j_{1}-1\right) \delta\right]\left[1+\left(j_{2}-1\right) \delta\right]\left[1+\left(j_{3}-1\right) \delta\right]\right)^{n}}{\left|v_{j_{1}} v_{j_{2}} v_{j_{3}}\right|}+\cdots \\
& \left.+\beta^{k-2} \prod_{j=2}^{k-1} \frac{[1+(j-1) \delta]^{n}}{\left|v_{j}\right|}\right\},
\end{aligned}
$$

where $\beta=2(1-\alpha)$ and $v_{k}=[1+(k-1) \delta]^{m}-[1+(k-1) \delta]^{n}$.

Proof. Define the function $p(z)$ by

$$
p(z)=\frac{1}{1-\alpha}\left(\frac{D^{m} f(z)}{D^{n} f(z)}-\alpha\right)=1+\sum_{j=1}^{\infty} c_{j} z^{j}
$$

Since $p(z)$ is the Carathéodory function, we have that

$$
\left|c_{j}\right| \leq 2 \quad(j=1,2,3, \ldots) .
$$

The definition of $p(z)$ implies that

$$
\frac{1}{(1-\alpha)}\left(D^{m} f(z)-\alpha D^{n} f(z)\right)=D^{n} f(z)\left(1+\sum_{j=1}^{\infty} c_{j} z^{j}\right)
$$


Since

$$
D^{n} f(z)=z+\sum_{j=2}^{\infty}[1+(j-1) \delta]^{n} a_{j} z^{j} \quad\left(n \in \mathbb{N}_{0}\right)
$$

we have

$$
\begin{aligned}
\frac{D^{m} f(z)-\alpha D^{n} f(z)}{1-\alpha}= & z+\frac{(1+\delta)^{m}-\alpha(1+\delta)^{n}}{1-\alpha} a_{2} z^{2}+\frac{(1+2 \delta)^{m}-\alpha(1+2 \delta)^{n}}{1-\alpha} a_{3} z^{3}+\cdots \\
& +\frac{[1+(k-1) \delta]^{m}-\alpha[1+(k-1) \delta]^{n}}{1-\alpha} a_{k} z^{k}+\cdots, \\
D^{n} f(z)\left(1+\sum_{j=1}^{\infty} c_{j} z^{j}\right)= & \left(z+\sum_{j=2}^{\infty}[1+(j-1) \delta]^{n} a_{j} z^{j}\right)\left(1+c_{1} z+\cdots+c_{k} z^{k}+\cdots\right) .
\end{aligned}
$$

Therefore, (2.11) shows that

$$
\begin{gathered}
z+\frac{(1+\delta)^{m}-\alpha(1+\delta)^{n}}{1-\alpha} a_{2} z^{2}+\frac{(1+2 \delta)^{m}-\alpha(1+2 \delta)^{n}}{1-\alpha} a_{3} z^{3}+\cdots+\frac{[1+(k-1) \delta]^{m}-\alpha[1+(k-1) \delta]^{n}}{1-\alpha} a_{k} z^{k}+\cdots \\
=\left(z+\sum_{j=2}^{\infty}[1+(j-1) \delta]^{n} a_{j} z^{j}\right)\left(1+c_{1} z+\cdots+c_{k} z^{k}+\cdots\right)
\end{gathered}
$$

If we consider the coefficients of $z^{k}$ of the both sides in the above equality, then we find that

$$
\left(\frac{[1+(k-1) \delta]^{m}-\alpha[1+(k-1) \delta]^{n}}{1-\alpha}-[1+(k-1) \delta]^{n}\right) a_{k}=\sum_{j=1}^{k-1}[1+(k-j-1) \delta]^{n} a_{k-j} c_{j}
$$

Therefore,

$$
\begin{aligned}
\left|a_{k}\right| & =\frac{1-\alpha}{\left|[1+(k-1) \delta]^{m}-[1+(k-1) \delta]^{n}\right|}\left|\sum_{j=1}^{k-1}[1+(k-j-1) \delta]^{n} a_{k-j} c_{j}\right| \\
& \leq \frac{1-\alpha}{\left|[1+(k-1) \delta]^{m}-[1+(k-1) \delta]^{n}\right|}\left(\sum_{j=1}^{k-1}[1+(k-j-1) \delta]^{n}\left|a_{k-j}\right|\left|c_{j}\right|\right) \\
& \leq \frac{2(1-\alpha)}{\left|[1+(k-1) \delta]^{m}-[1+(k-1) \delta]^{n}\right|}\left(\sum_{j=1}^{k-1}[1+(k-j-1) \delta]^{n}\left|a_{k-j}\right|\right),
\end{aligned}
$$


since $\left|c_{j}\right| \leq 2(j=1,2,3 \ldots)$. Thus, for $\beta=2(1-\alpha)$ and $v_{k}=[1+(k-1) \delta]^{m}-[1+(k-1) \delta]^{n}$, we obtain

$$
\begin{aligned}
& \left|a_{k}\right| \leq \beta \frac{1}{\left|v_{k}\right|}\left\{1+(1+\delta)^{n} \frac{\beta}{\left|v_{2}\right|}+(1+2 \delta)^{n} \frac{\beta}{\left|v_{3}\right|}+(1+3 \delta)^{n} \frac{\beta}{\left|v_{4}\right|}+\cdots+(1+(k-2) \delta)^{n} \frac{\beta}{\left|v_{k-1}\right|}\right. \\
& +(1+\delta)^{n}(1+2 \delta)^{n} \frac{\beta^{2}}{\left|v_{2} v_{3}\right|}+(1+\delta)^{n}(1+3 \delta)^{n} \frac{\beta^{2}}{\left|v_{2} v_{4}\right|} \\
& +(1+\delta)^{n}(1+4 \delta)^{n} \frac{\beta^{2}}{\left|v_{2} v_{5}\right|}+\cdots+(1+\delta)^{n}(1+(k-2) \delta)^{n} \frac{\beta^{2}}{\left|v_{2} v_{k-1}\right|} \\
& +(1+2 \delta)^{n}(1+3 \delta)^{n} \frac{\beta^{2}}{\left|v_{3} v_{4}\right|}+(1+2 \delta)^{n}(1+4 \delta)^{n} \frac{\beta^{2}}{\left|v_{3} v_{5}\right|}+\cdots \\
& +(1+2 \delta)^{n}(1+(k-2) \delta)^{n} \frac{\beta^{2}}{\left|v_{3} v_{k-1}\right|}+\cdots \\
& +(1+\delta)^{n}(1+2 \delta)^{n}(1+3 \delta)^{n} \frac{\beta^{3}}{\left|v_{2} v_{3} v_{4}\right|}+(1+\delta)^{n}(1+3 \delta)^{n}(1+4 \delta)^{n} \frac{\beta^{3}}{\left|v_{2} v_{4} v_{5}\right|}+\cdots \\
& \left.+(1+\delta)^{n}(1+(k-3) \delta)^{n}(1+(k-2) \delta)^{n} \frac{\beta^{3}}{\left|v_{2} v_{k-2} v_{k-1}\right|}+\beta^{k-2} \prod_{j=2}^{k-1} \frac{[1+(j-1) \delta]^{n}}{\left|v_{j}\right|}\right\} \\
& =\frac{\beta}{\left|v_{k}\right|}\left\{1+\beta \sum_{j=2}^{k-1} \frac{[1+(j-1) \delta]^{n}}{\left|v_{j}\right|}+\beta^{2} \sum_{j_{2}>j_{1}}^{k-1} \sum_{j_{1}=2}^{k-2} \frac{\left(\left[1+\left(j_{1}-1\right) \delta\right]\left[1+\left(j_{2}-1\right) \delta\right]\right)^{n}}{\left|v_{j_{1}} v_{j_{2}}\right|}\right. \\
& \left.+\beta^{3} \sum_{j_{3}>j_{2}}^{k-1} \sum_{j_{2}>j_{1}}^{k-2} \sum_{j_{1}=2}^{k-3} \frac{\left(\left[1+\left(j_{1}-1\right) \delta, 1+\left(j_{2}-1\right) \delta, 1+\left(j_{3}-1\right) \delta\right]\right)^{n}}{\left|v_{j_{1}} v_{j_{2}} v_{j_{3}}\right|}+\cdots+\beta^{k-2} \prod_{j=2}^{k-1} \frac{[1+(j-1) \delta]^{n}}{\left|v_{j}\right|}\right\} .
\end{aligned}
$$

This completes the proof of Theorem 2.3.

If we take $\delta=1$ in Theorems 2.1 and 2.3, we can get the results due to Sümer Eker and Owa [3].

\section{Extreme points}

In view of Theorem 2.1, we now introduce the subclass $\widetilde{\mathcal{S}}_{m, n, \delta}(\alpha) \subset \mathcal{S}_{m, n, \delta}(\alpha)$, which consists of function

$$
f(z)=z+\sum_{j=2}^{\infty} a_{j} z^{j} \quad\left(a_{j} \geq 0\right)
$$

whose Taylor-Maclaurin coefficients satisfy inequality (2.1). Now, let us determine extreme points of the class $\widetilde{\mathcal{S}}_{m, n, \delta}(\alpha)$. 
Theorem 3.1. Let $f_{1}(z)=z$ and

$$
f_{j}(z)=z+\frac{2(1-\alpha)}{\Psi(m, n, j, \delta, \alpha)} z^{j} \quad(j=2,3, \ldots),
$$

where $\Psi(m, n, j, \delta, \alpha)$ is given by (2.2).

Then $f \in \widetilde{S}_{m, n}(\alpha)$ if and only if it can be expressed in the form

$$
f(z)=\sum_{j=1}^{\infty} \eta_{j} f_{j}(z)
$$

where $\eta_{j}>0$ and $\sum_{j=1}^{\infty} \eta_{j}=1$.

Proof. Suppose that

$$
f(z)=\sum_{j=1}^{\infty} \eta_{j} f_{j}(z)=z+\sum_{j=2}^{\infty} \eta_{j} \frac{2(1-\alpha)}{\Psi(m, n, j, \delta, \alpha)} z^{j}
$$

Then

$$
\sum_{j=2}^{\infty} \Psi(m, n, j, \delta, \alpha) \frac{2(1-\alpha)}{\Psi(m, n, j, \delta, \alpha)} \eta_{j}=2(1-\alpha) \sum_{j=2}^{\infty} \eta_{j}=2(1-\alpha)\left(1-\eta_{1}\right)<2(1-\alpha),
$$

which shows that $f$ satisfies condition (2.1) and therefore $f \in \widetilde{\mathcal{S}}_{m, n, \delta}(\alpha)$.

Conversely, suppose that $f \in \widetilde{\mathcal{S}}_{m, n, \delta}(\alpha)$. Since

$$
a_{j} \leq \frac{2(1-\alpha)}{\Psi(m, n, j, \delta, \alpha)} \quad(j=2,3, \ldots),
$$

we may set

$$
\begin{aligned}
& \eta_{j}=\frac{\Psi(m, n, j, \delta, \alpha)}{2(1-\alpha)} a_{j} \\
& \eta_{1}=1-\sum_{j=2}^{\infty} \eta_{j} .
\end{aligned}
$$

Then we obtain

$$
f(z)=\sum_{j=1}^{\infty} \eta_{j} f_{j}(z)
$$

which completes the proof of Theorem 3.1.

Corollary 3.2. The extreme points of $\widetilde{\mathcal{S}}_{m, n, \delta}(\alpha)$ are the functions $f_{1}(z)=z$ and

$$
f_{j}(z)=z+\frac{2(1-\alpha)}{\Psi(m, n, j, \delta, \alpha)} z^{j} \quad(j=2,3, \ldots),
$$

where $\Psi(m, n, j, \delta, \alpha)$ is given by (2.2). 


\section{Integral means inequalities for fractional derivative}

We will make use of the following definitions of fractional derivatives by Owa [4], and Srivastava and Owa [5].

Definition 4.1. The fractional derivative of order $\lambda$ is defined, for a function $f$, by

$$
D_{z}^{\lambda} f(z)=\frac{1}{\Gamma(1-\lambda)} \frac{d}{d z} \int_{0}^{z} \frac{f(\xi)}{(z-\xi)^{\lambda}} d \xi \quad(0 \leq \curlywedge<1)
$$

where $f$ is an analytic function in a simply connected region of $z$-plane containing the origin, and the multiplicity of $(z-\xi)^{-\lambda}$ is removed by requiring $\log (z-\xi)$ to be real when $z-\xi>0$.

Definition 4.2. Under the hypotheses of Definition 4.1, the fractional derivative of order $p+\lambda$ is defined, for a function $f$, by

$$
D_{z}^{p+\lambda} f(z)=\frac{d^{p}}{d z^{p}} D_{z}^{\lambda} f(z) \quad\left(0 \leq \lambda<1 ; p \in \mathbb{N}_{0}\right)
$$

It readily follows from $(4.1)$ that

$$
D_{z}^{\lambda} z^{k}=\frac{\Gamma(k+1)}{\Gamma(k-\lambda+1)} z^{k-\lambda} \quad(0 \leq \lambda<1, k \in \mathbb{N})
$$

Further, we need the concept of subordination between analytic functions [6] and a subordination theorem of Littlewood in our investigation.

Definition 4.3. For two functions $f$ and $g$, analytic in $\mathbb{U}$, say that the function $f(z)$ is subordinate to $g(z)$ in $\mathbb{U}$, and write

$$
f(z) \prec g(z) \quad(z \in \mathbb{U})
$$

if there exists a Schwarz function $w(z)$, analytic in $\mathbb{U}$ with $w(0)=0$ and $|w(z)|<1$ such that

$$
f(z)=g(w(z)) \quad(z \in \mathbb{U})
$$

In particular, if the function $g$ is univalent in $\mathbb{U}$, the above subordination is equivalent to

$$
f(0)=g(0), \quad f(\mathbb{U}) \subset g(\mathbb{U})
$$

In 1925, Littlewood [7] proved the following subordination theorem.

Lemma 4.4. If $f(z)$ and $g(z)$ are analytic in $\mathbb{U}$ with $f(z)<g(z)$, then for $\mu>0$ and $z=r e^{i \theta}(0<r<1)$,

$$
\int_{0}^{2 \pi}|f(z)|^{\mu} d \theta \leqq \int_{0}^{2 \pi}|g(z)|^{\mu} d \theta
$$


Theorem 4.5. Let $f(z) \in \widetilde{\mathcal{S}}_{m, n, \delta}(\alpha)$ and suppose that

$$
\sum_{j=2}^{\infty}(j-p)_{p+1} a_{j} \leq \frac{2(1-\alpha) \Gamma(k+1) \Gamma(3-\lambda-p)}{\Psi(m, n, k, \delta, \alpha) \Gamma(k+1-\lambda-p) \Gamma(2-p)}
$$

for some $j \geq p, 0 \leq \lambda<1$, and $(j-p)_{p+1}$ denote the Pochhammer symbol defined by $(j-p)_{p+1}=$ $(j-p)(j-p+1) \cdots j$. Also let the function

$$
f_{k}(z)=z+\frac{2(1-\alpha)}{\Psi(m, n, k, \delta, \alpha)} z^{k} \quad(k \geq 2)
$$

If there exists an analytic function $w(z)$ given by

$$
(w(z))^{k-1}=\frac{\Psi(m, n, k, \delta, \alpha) \Gamma(k+1-\lambda-p)}{2(1-\alpha) \Gamma(k+1)} \sum_{j=2}^{\infty}(j-p)_{p+1} \frac{\Gamma(j-p)}{\Gamma(j+1-\lambda-p)} a_{j} z^{j-1}, \quad(k \geq p),
$$

then for $z=r e^{i \theta}$ and $0<r<1$,

$$
\int_{0}^{2 \pi}\left|D_{z}^{p+\lambda} f(z)\right|^{\mu} d \theta \leq \int_{0}^{2 \pi}\left|D_{z}^{p+\lambda} f_{k}(z)\right|^{\mu} d \theta \quad(0 \leq \lambda<1, \mu>0) .
$$

Proof. By virtue of the fractional derivative formula (4.3) and Definition 4.2, we find from (3.1) that

$$
\begin{aligned}
D_{z}^{p+\lambda} f(z) & =\frac{z^{1-\lambda-p}}{\Gamma(2-\lambda-p)}\left\{1+\sum_{j=2}^{\infty} \frac{\Gamma(2-\lambda-p) \Gamma(j+1)}{\Gamma(j+1-\lambda-p)} a_{j} z^{j-1}\right\} \\
& =\frac{z^{1-\lambda-p}}{\Gamma(2-\lambda-p)}\left\{1+\sum_{j=2}^{\infty} \Gamma(2-\lambda-p)(j-p)_{p+1} \Phi(j) a_{j} z^{j-1}\right\},
\end{aligned}
$$

where

$$
\Phi(j)=\frac{\Gamma(j-p)}{\Gamma(j+1-\lambda-p)} \quad(0 \leq \lambda<1 ; j \geq p) .
$$

Since $\Phi(j)$ is a decreasing function of $j$, we have

$$
0<\Phi(j) \leq \Phi(2)=\frac{\Gamma(2-p)}{\Gamma(3-\lambda-p)}
$$

Similarly, from (4.3), (4.9), and Definition 4.2, we obtain

$$
D_{z}^{p+\lambda} f_{k}(z)=\frac{z^{1-\lambda-p}}{\Gamma(2-\lambda-p)}\left\{1+\frac{2(1-\alpha) \Gamma(2-\lambda-p) \Gamma(k+1)}{\Psi(m, n, k, \delta, \alpha) \Gamma(k+1-\lambda-p)} z^{k-1}\right\}
$$


For $z=r e^{i \theta}, 0<r<1$, we must show that

$$
\begin{aligned}
\int_{0}^{2 \pi} \mid & +\left.\sum_{j=2}^{\infty} \Gamma(2-\lambda-p)(j-p)_{p+1} \Phi(j) a_{j} z^{j-1}\right|^{\mu} d \theta \\
& \leq \int_{0}^{2 \pi}\left|1+\frac{2(1-\alpha) \Gamma(2-\lambda-p) \Gamma(k+1)}{\Psi(m, n, k, \delta, \alpha) \Gamma(k+1-\lambda-p)} z^{k-1}\right|^{\mu} d \theta \quad(\mu>0) .
\end{aligned}
$$

Thus by applying Littlewood's subordination theorem, it would be suffice to show that

$$
1+\sum_{j=2}^{\infty} \Gamma(2-\lambda-p)(j-p)_{p+1} \Phi(j) a_{j} z^{j-1} \prec 1+\frac{2(1-\alpha) \Gamma(2-\lambda-p) \Gamma(k+1)}{\Psi(m, n, k, \delta, \alpha) \Gamma(k+1-\lambda-p)} z^{k-1}
$$

By setting

$$
1+\sum_{j=2}^{\infty} \Gamma(2-\lambda-p)(j-p)_{p+1} \Phi(j) a_{j} z^{j-1}=1+\frac{2(1-\alpha) \Gamma(2-\lambda-p) \Gamma(k+1)}{\Psi(m, n, k, \delta, \alpha) \Gamma(k+1-\lambda-p)} w(z)^{k-1}
$$

we find that

$$
(w(z))^{k-1}=\frac{\Psi(m, n, k, \delta, \alpha) \Gamma(k+1-\lambda-p)}{2(1-\alpha) \Gamma(k+1)} \sum_{j=2}^{\infty}(j-p)_{p+1} \Phi(j) a_{j} z^{j-1}
$$

which readily yields $w(0)=0$. Further, we prove that the analytic function $w(z)$ satisfies $|w(z)|<1, z \in \mathbb{U}$ for (4.10). We know that

$$
\begin{aligned}
|w(z)|^{k-1} & \leq\left|\frac{\Psi(m, n, k, \delta, \alpha) \Gamma(k+1-\lambda-p)}{2(1-\alpha) \Gamma(k+1)} \sum_{j=2}^{\infty}(j-p)_{p+1} \Phi(j) a_{j} z^{j-1}\right| \\
& \leq \frac{\Psi(m, n, k, \delta, \alpha) \Gamma(k+1-\lambda-p)}{2(1-\alpha) \Gamma(k+1)} \sum_{j=2}^{\infty}(j-p)_{p+1} \Phi(j) a_{j}|z|^{j-1} \\
& \leq|z| \frac{\Psi(m, n, k, \delta, \alpha) \Gamma(k+1-\lambda-p)}{2(1-\alpha) \Gamma(k+1)} \Phi(2) \sum_{j=2}^{\infty}(j-p)_{p+1} a_{j} \\
& =|z| \frac{\Psi(m, n, k, \delta, \alpha) \Gamma(k+1-\lambda-p)}{2(1-\alpha) \Gamma(k+1)} \frac{\Gamma(2-p)}{\Gamma(3-\lambda-p)} \sum_{j=2}^{\infty}(j-p)_{p+1} a_{j} \\
& \leq|z|<1
\end{aligned}
$$

by means of the hypothesis of Theorem 4.5.

As special case $p=0$, Theorem 4.5 readily yields.

Corollary 4.6. Let $f(z) \in \widetilde{\mathcal{S}}_{m, n, \delta}(\alpha)$ and suppose that

$$
\sum_{j=2}^{\infty} j a_{j} \leq \frac{2(1-\alpha) \Gamma(k+1) \Gamma(3-\lambda)}{\Psi(m, n, k, \delta, \alpha) \Gamma(k+1-\lambda)}
$$


for some $0 \leq \lambda<1$. Also let the function

$$
f_{k}(z)=z+\frac{2(1-\alpha)}{\Psi(m, n, k, \delta, \alpha)} z^{k} \quad(k \geq 2) .
$$

If there exists an analytic function $w(z)$ given by

$$
(w(z))^{k-1}=\frac{\Psi(m, n, k, \delta, \alpha) \Gamma(k+1-\lambda)}{2(1-\alpha) \Gamma(k+1)} \sum_{j=2}^{\infty} \frac{\Gamma(j+1)}{\Gamma(j+1-\lambda)} a_{j} z^{j-1},
$$

then for $z=r e^{i \theta}$ and $0<r<1$,

$$
\int_{0}^{2 \pi}\left|D_{z}^{\lambda} f(z)\right|^{\mu} d \theta \leq \int_{0}^{2 \pi}\left|D_{z}^{\lambda} f_{k}(z)\right|^{\mu} d \theta \quad(0 \leq \lambda<1, \mu>0) .
$$

\section{Acknowledgment}

The authors are thankful to the referees for their comments and suggestions.

\section{References}

[1] F. M. Al-Oboudi, "On univalent functions defined by a generalized Sălăgean operator," International Journal of Mathematics and Mathematical Sciences, vol. 2004, no. 27, pp. 1429-1436, 2004.

[2] G. S. Sălăgean, "Subclasses of univalent functions," in Complex Analysis—5th Romanian-Finnish seminar, Part 1 (Bucharest, 1981), vol. 1013 of Lecture Notes in Mathematics, pp. 362-372, Springer, Berlin, Germany, 1983.

[3] S. Sümer Eker and S. Owa, "New applications of classes of analytic functions involving the Sălăgean operator," in Proceedings of the International Symposium on Complex Function Theory and Applications, pp. 21-34, Transilvania University of Printing House, Braşov, Romania, September 2006.

[4] S. Owa, "On the distortion theorems. I," Kyungpook Mathematical Journal, vol. 18, no. 1, pp. 53-59, 1978.

[5] H. M. Srivastava and S. Owa, Eds., Univalent Functions, Fractional Calculus, and Their Applications, Ellis Horwood Series: Mathematics and Its Applications, Ellis Horwood, Chichester, UK, 1989.

[6] P. L. Duren, Univalent Functions, Springer, New York, NY, USA, 1983.

[7] J. E. Littlewood, "On inequalities in the theory of functions," Proceedings of the London Mathematical Society, vol. 23, no. 1, pp. 481-519, 1925. 